\title{
Mass-IVR - A High Performance Outbound Interactive Voice Response Management System
}

\author{
Hüseyin Abanoz and Özgür Erbaş
}

\begin{abstract}
Organizations need to announce activities (e.g meetings), run polls, give information, gather feedback about their business and make advertisements. With the improvements in IVR technology, fixed \& mobile phones create a great opportunity to meet those needs. Mass-IVR System was designed with those facts and necessities as motive and it provides the ability to call millions of telecom subscribers, let them listen a designated voice message and collect their feedbacks in the form of Dual Tone Multi-Frequency (DTMF) codes.
\end{abstract}

Index Terms-IVR, mass calls, DTMF, announcement, optimization.

\section{INTRODUCTION}

Organizations need to announce activities (e. g meetings), run polls, give information, gather feedback about their business and make advertisements. There are two kinds of IVR systems [1]. Inbound version of IVR systems are called by users to request information or run different types of transactions. Outbound version of IVR, on the other hand, calls users to conduct surveys, announce events etc.

IVR has numerous advantages over other means of communication. Cost per call is likely to be low, accuracy is improved and response rates are high [2]-[5].

The main motivation behind proposed Mass-IVR System is lack of a solution which is capable of transmitting mass calls to large numbers of audiences within limited time spans while high performance expectations are satisfied. Proposed Mass-IVR system is capable of establishing calls to targeted audiences, and in the meantime it respects the capacities of the resources used and utilizes those resources efficiently while keeping the performance high. Furthermore; load, which Media Gateway Controller (MGC) - one of the software layer component of Mass-IVR-servers can carry, and voice trunks, which goes to the targeted areas, are among the most important resources so they should be utilized efficiently. Another motive is the durability of the system. Server breakdowns are among the major problems which affects durability of the systems. Therefore; all physical equipments, which are part of Mass-IVR hardware layer, has redundancy. If one server breaks down, corresponding redundant server will assure the continuation of the service

Manuscript received October 13, 2014; revised May 12, 2015. This work is supported by the Alcatel-Lucent R\&D Department.

Hüseyin Abanoz is with the Alcatel-Lucent, Turkey (tel.: +90-216-579-2080; fax: +90-216-364-1085; e-mail: huseyin.abanoz@alcatel-lucent.com).

Özgür Erbaş is with the Dept. of Computer Engineering, Boğaziçi University, Istanbul and Alcatel-Lucent, Turkey (tel.: +90-216-579-2896; fax: +90-216-364-1085; e-mail: ozgur.erbas@ alcatel-lucent.com). without outage.

As a result, Mass-IVR solution has been created. Solution system architecture consists of two fundamental components which include Mass-IVR Software Layer and Mass-IVR Hardware Layer as shown in Fig. 1.

Hardware Layer consists of Mass-IVR service servers, Media Resource Function (MRF) and Media Gateway Controller.

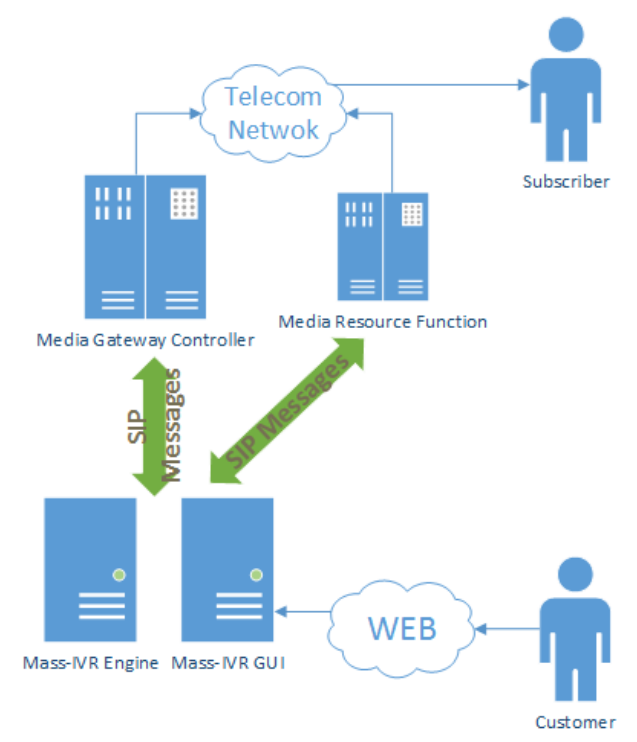

Fig. 1. Mass-IVR system hardware layer.

Furthermore, there are two important software components in Mass-IVR Software Layer which are: Mass-IVR Portal and Mass-IVR Engine. Mass-IVR Portal is a web application that allows users to arrange and manage campaigns through internet. In order to increase the efficiency of the solution and to automate the process of campaign creation, a Web Service interface is also provided. Secondly, Mass-IVR Engine Service is a software service which executes campaigns that are created through portal. Basically, engine service manages lifecycle of the campaigns and creates result reports. It was designed to assure completion of campaigns that have large sets of subscriber numbers, within the desired time spans.

In Sections II and III, the details of the proposed system model and its key points are presented. Next sections will cover Hardware and Software Layers of the solution respectively.

\section{MASS-IVR HARDWARE LAYER}

Mass-IVR Hardware Layer is composed of Mass-IVR service servers, MRF servers and MGC servers.

First component is MGC Server and it is used to perform launching, forwarding, updating and terminating calls 
requests in the telecom network which are initiated by engine service. MGC server is also named as Soft Switch. If connected telecom network employs a messaging protocol other than SIP [6] then required message conversions are performed by MGC Servers.

For each launched call, messages come to MGC server first, after being processed by MGC server then comes to Mass-IVR engine service. Message processing includes required conversions, updating call state data, management of timers related to messaging. Tens of calls are launched in a second and generally MGC servers are not dedicated to Mass-IVR service and have other responsibilities. In other words, MGC servers run under heavy load. Therefore; capacity of MGC servers is among the most important limited resources for Mass-IVR systems.

Second component is MRF Server and its responsibilities are to connect the calls as remote party, which were launched by Mass-IVR Engine service, to play the designated voice message that is wished to announce to subscribers and to inform Mass-IVR Engine service about the digits dialed by the subscriber.

Note that, in order for MRF servers to fulfill their tasks, the voice messages, which need to be played, should be uploaded to MRF servers before the campaign, to which the voice messages belong, starts. MRF servers can only play announcement files which are encoded with one of the codecs, like G711A, installed to MRF servers. Additionally, in order to avoid any further problem, it may be required to convert voice message files into correct format before they are used by MRF servers.

Furthermore, capacity of the MRF servers is among the scarce resources so Mass-IVR system should utilize it efficiently. While evaluating resource utilization, calls attempted per second as well as length of the voice messages should be taken into consideration. Since voice messages with short durations are completed quickly and release used resources for use of other calls, it should be preferred to keep voice message duration short.

Last important component of the hardware layer is Mass-IVR Server which hosts all the applications that Mass-IVR service uses. Required database support for engine service and portal could be deployed into separate servers or same server if it has enough computing power (CPU) and memory. In addition; having a single server for engine service application and database could be considered as a preferable idea because network latency while reaching database node becomes minimum in such configuration.

For security reasons, Mass-IVR servers need to be behind a firewall. They also need to have access to other Mass-IVR servers such as MGC and MRF servers so required rules and access must be provided. Furthermore, in such cases that MRF and MGC servers reside in different networks, according to configuration, Mass-IVR service servers may need more than one network interface.

Note that, there might be multiple Mass-IVR, MGC and MRF servers or there might be a single MRF server that is provided with redundancy.

\section{MASS-IVR SOFTWARE LAYER}

Mass-IVR software layer has two major components which includes; Mass-IVR Web Portal and Mass-IVR Engine service.

First component is Mass-IVR Portal which is used to create and manage campaigns through internet. Simply, a campaign is a task in Mass-IVR terminology which consists of a voice message, set of numbers to call, a schedule to realize the calls and some other meta-data. In a common scenario, users upload a voice message file, specify a set of subscriber numbers (target audience), determine the schedule for arranging calls and finalize the creation of the campaign. Once a campaign is created, it could be launched immediately or scheduled for another time. Note that, after a campaign is started, it could be paused temporally for any reason or can be cancelled completely. After all subscriber numbers belonging to a campaign are called, the campaign is marked as completed. Result of the campaign can be viewed as a report via portal. In addition, report(s) can be downloaded for data analysis and future studies. Note that, web service is also provided to make it possible to automate campaign creation. With the ability of machine to machine interaction, through the web service capability, it becomes possible to create campaigns using third party applications.

Second component is Mass-IVR Engine Service. Basically, engine service realizes campaigns that are created through portal or web service. Engine service is one of the most important components of the Mass-IVR system. It resides in the heart of the Mass-IVR system. Engine service needs to perform well such that it can initiate as many calls as possible and be smart enough to respect the capacity of the resources utilized. Mass-IVR Engine service has been designed in this way such that once a campaign is created; it is started by engine service. Number list is fetched from database and each call is launched in compliance with existing limits. To fetch numbers from database, engine service uses a memory buffer. Launched numbers are removed from this fetch buffer and put to active calls buffer. After exhaustion of numbers, fetch buffer is re-filled with numbers from DB. It is ensured that all active campaigns benefits from fetch buffer equally.

\section{Mass-IVR CALl SEQUENCE}

Launching a call contains several steps;

1) Subscriber number is called. A SIP invite request is sent to MGC servers. MGC servers convert the message to format of the underlying telecom network if required and initialize a call in telecom network. When Subscriber opens her phone, first leg of the IVR call is said to be established.

2) MRF is called. A SIP invite request is sent to MRF server. When MRF server accepts the call second leg of the IVR call is said to be established.

3) Session information from MRF call needs to be transferred to subscriber call. Thus a second SIP invite is sent to MGC and MGC re-transmits session information to subscriber call.

4) Eventually an IVR call is launched.

Once a call is launched, it is proceeded and a voice message transmission phase starts. In order to start voice message transmission, a SIP info message is sent to MRF server. When info message containing play request arrives, MRF server starts voice message transmission and waits for 
user inputs. After the end of the voice message, digits dialed by the subscriber sent from MRF server to engine service by a second sip INFO message. Whole process is depicted as a sequence diagram in Fig. 2.

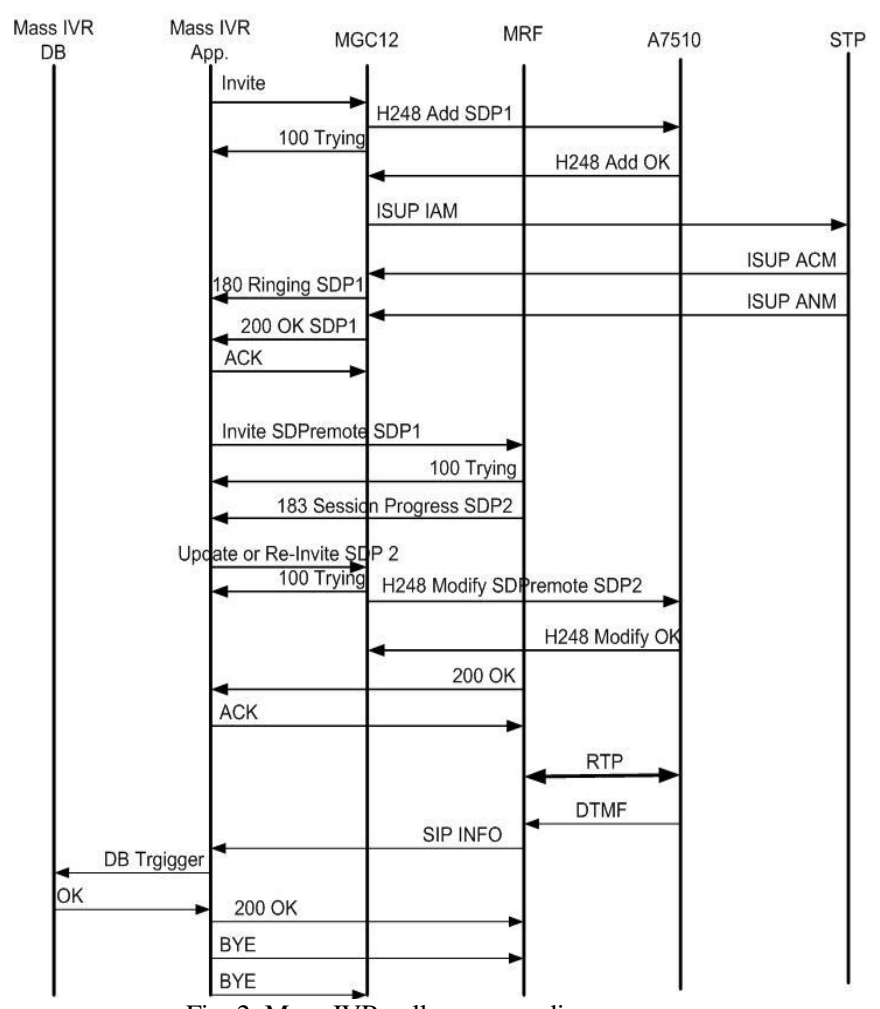

Fig. 2. Mass-IVR call sequence diagram.

\section{RESULTS}

A call is evaluated in following ways and categorized accordingly. If a call fails because of an application or network error or some other cause, this call is said to be a Failed Call. If call attempt succeeds and subscriber phone rings but the phone is not opened because the subscriber is busy or because the subscriber does not want to answer the call for some reason, this call is said to be a Not Replied Call. If everything goes fine and user accepts the call, this call is said to be a Successful Call.

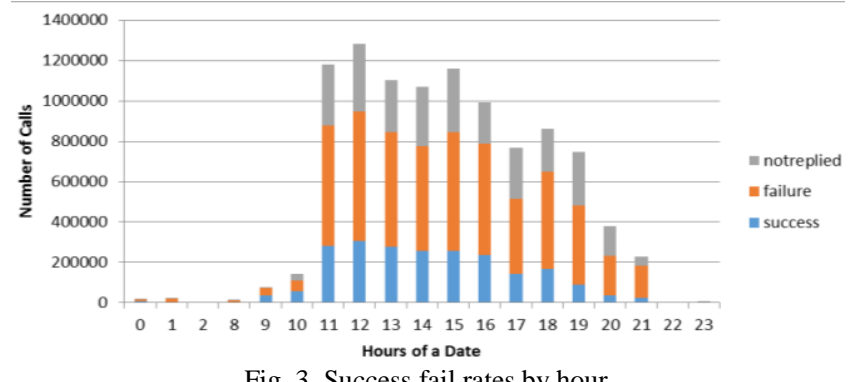

Fig. 3 gives distribution of successful, failed and not replied calls by hours of a day. Fig. 4 displays the same data with vertical axis showing the percentage of successful, failed and not replied calls in a specific hour.

When the charts in Fig. 3 and Fig. 4 are interpreted it is observed that 9 and 10 o'clock have high success percentage but low number of calls. Thus, number of calls made at those hours may be increased to positively affect overall success of campaigns

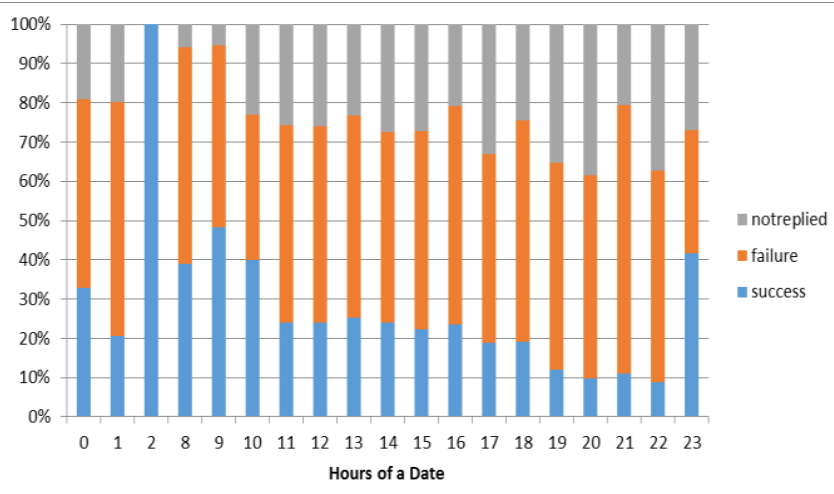

Fig. 4. Success fail percentage rates by hour.

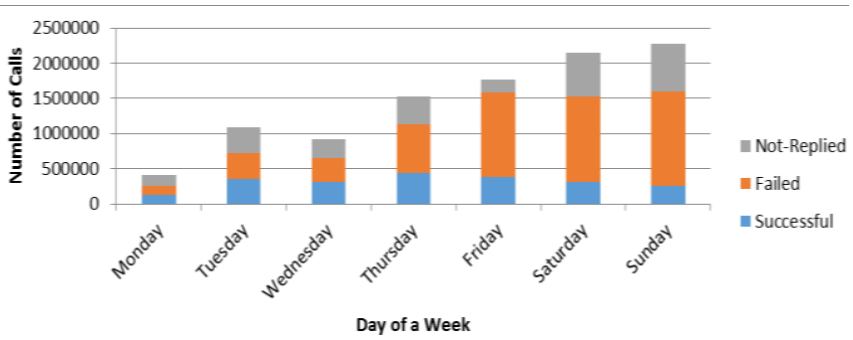

Fig. 5. Daily success-fail rates.

Fig. 5 shows successful, failed and not-replied calls by days of a week. When examined, it is seen that Monday has a good success-fail rate but it is not preferred in past campaigns. To enhance overall success of campaigns use of Monday can be promoted.

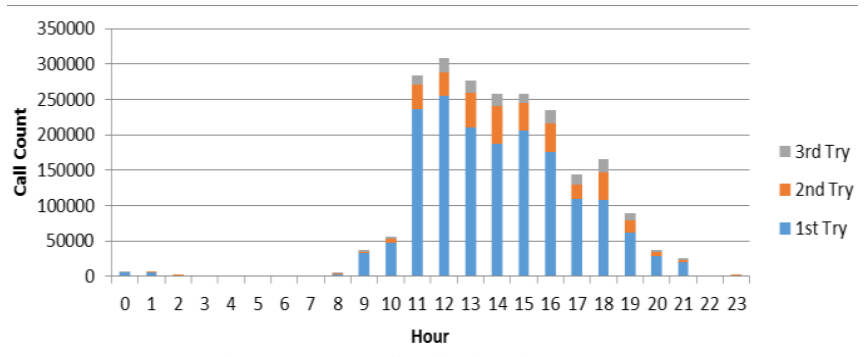

Fig. 6. Success distribution by try count.

Fig. 6 depicts the distribution of successful calls by try count. Most of the calls reach to success at first try. Much less of the failed-at-first calls succeed at second try. Successful calls occurring at third attempt are even less. This might be an indicator of a better scheduling algorithm to reach those users.

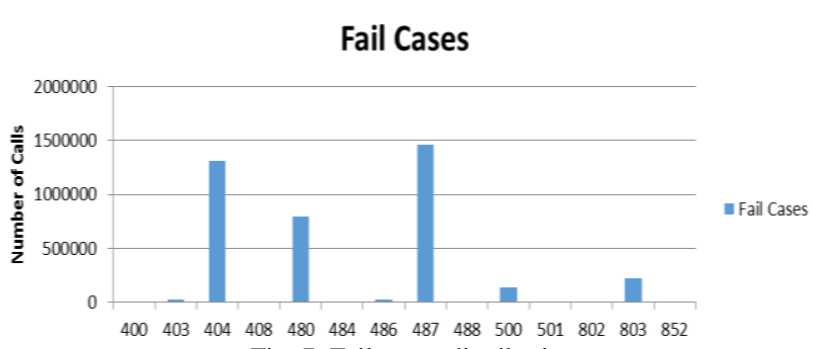

Fig. 7. Fail cause distribution.

Fig. 7 displays the distribution of failed calls by cause. $4 \mathrm{XX}$ and $5 \mathrm{XX}$ codes are SIP response codes (For a complete list of SIP response codes see [4]). 8XX codes are non-standard and their meanings are given in Table I. 
TABLE I: COMMON SIP RESPONSE CODES

\begin{tabular}{ll}
\hline \hline Response Code & Meaning \\
\hline 802 & MGC Timeout \\
803 & Ringing Timeout \\
852 & User Ended \\
XXX & Please see [4] \\
\hline
\end{tabular}

Major failure cases are: Not Found, Temporarily Unavailable and Request Terminated. These points require investigation and effort to minimize those failures.

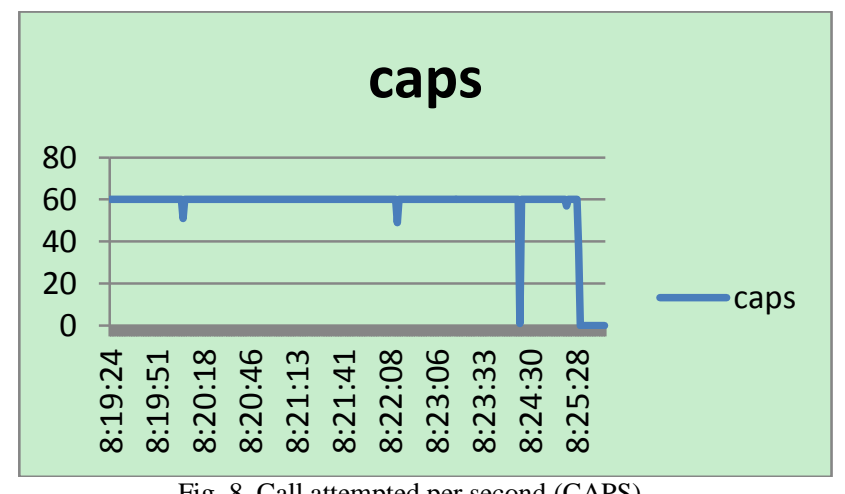

Fig. 8 is the chart of Call Attempted per Seconds aka CAPS. Mass-IVR system can attempt 60 calls per second, which gives the power to call about 18 million subscribers in a week. Falls in the chart correspond to the times when numbers in buffer are depleted and new numbers are being fetched.

\section{CONCLUSION}

Mass-IVR system is designed and implemented according to the points described above. Current solution is up \& running in production environment and total numbers called in different campaigns have already reached to 15 million.

As a future work, it is planned to improve the system in terms of security. For instance, Portal login procedure will be improved to prevent attacks like XSRF. HTTP headers will be filtered in a way which does not reveal sensitive information. Authorization procedure will be enhanced to assure authorized access to confidential data. Additionally portal message transmissions are planned to be made more secure. Furthermore, data mining studies will be done to improve service quality. Peak hours and hours that most successful feedbacks are collected may be determined dynamically to find best schedules to launch campaigns.

\section{REFERENCES}

[1] P. M. Couper, "The future of modes of data collection," Public Opinion Quarterly, vol. 75, no. 5, pp. 889-908, 2011.

[2] S. Fricker, M. Galesic, R. Tourangeau, and T. Yan, "An experimental comparison of web and telephone surveys," Public Opinion Quarterly, vol. 69 , no. 3, pp. 370-392, 2005 .

[3] R. Corkrey and L. Parkinson, "Interactive voice response: Review of studies 1989-2000," Behavior Research Methods, Instruments, \& Computers, vol. 34, no. 3, pp. 342-353, 2002.

[4] A. L. Holbrook, M. C. Green, and J. A. Krosnick, "Telephone versus face-to-face interviewing of national probability samples with long questionnaires," Public Opinion Quarterly, vol. 67, pp. 79-125.

[5] G. L. Rose, J. M. Skelley, G. J. Badger, C. D. Maclean, M. P. Malgeri, and J. E. Helzer, "Automated screening for at-risk drinking in a primary care office using interactive voice response," J Stud. Alcohol Drugs, vol. 71, no. 5, pp. 734-738, 2010.

[6] SIP: Session initiation protocol. The Internet Engineering Task Force (IETF®). [Online]. Available: https://www.ietf.org/rfc/rfc3261.txt

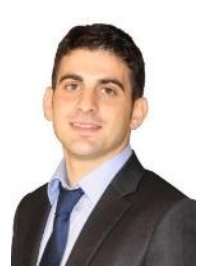

Hüseyin Abanoz was born in 1987 and grew up in Istanbul, Turkey. He completed his bachelor degree in computer engineering from Marmara University at Istanbul, Turkey in 2010 .

Currently, he is working as a software engineer in Alcatel-Lucent, Turkey since 2010 which is at Istanbul.

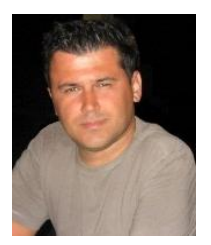

Özgür Erbaș was born in Nov. 1980. He grew up in Istanbul, Turkey. He completed elementary, middle and high schools in government schools. Then, he completed his bachelors degree in computer engineering from Marmara Universityand Isik University Istanbul, Turkey and his MSc degree also in computer engineering from Bogazici University. He is a student at the Bogazici University and currently is taking up a $\mathrm{PhD}$ degree in Computer Engineering Department. As a senior student he has been trained to perform highly in different fields such as parallel programming, resource management and grid computing.

He has worked in different industries including government, private initiations and banking. As soon as he graduated, he started working in Koc Holding as a software developer. After 1 year, he decided to continue his academic carrier and he went to Isik University. Then, he started working for Tubitak Ulakbim as a system admin while doing MSc in Bogazici University. After 3 years, he started to work in Garanti Bank as a software developer and he worked there for 3 years. Currently, he is the manager of OSS Assurance team in the company called Alcatel-Lucent which is a telco company. 